\title{
Intelligent High-Level RFID Event Transformation Utilising Non-Monotonic Reasoning
}

\author{
Peter Darcy, Bela Stantic and Abdul Sattar \\ Institute for Integrated and Intelligent Systems, Griffith University \\ Queensland, Australia \\ Email: \{P.Darcy, B.Stantic, A.Sattar $\} @$ griffith.edu.au
}

\begin{abstract}
Radio Frequency Identification technology utilises radio communication between tags and readers to automatically recognise large amounts of items reducing cost in both time and effort. Although there is a wide array of applications which RFID may be applied to, there are several issues that hinder the adoption of the technology into various commercial sectors. One of these problems is that the data recorded by readers are becoming increasingly difficult to decipher in its raw form resulting in complex spatio-temporal aspects. To counter the effects of this issue, we have proposed a concept that employs Clausal Defeasible Logic Non-Monotonic Reasoning to correctly identify a high-level event. From our experimental evaluation, we have shown that our approach surpasses the state-of-the-art probabilistic method in both identifying events and limiting falsepositive anomalies into the data set.
\end{abstract}

\section{INTRODUCTION}

Radio Frequency Identification (RFID) is an automated system consisting of tags that wirelessly communicate with readers in order to identify their locations with ease. Unfortunately, factors such as low level readings, errors, large volumes of intake data and complex spatio-temporal aspects prevent the system to be widely accepted among the commercial sector. One of the main problems associated with the data is the lowlevel observations that prevent the recorded data from being useful among different users.

Several probabilistic methodologies have been proposed in the past to transform these low-level observations into highlevel events. However, in the scenario in which high amounts of ambiguity is present, the integrity of the resulting events may be lowered. To combat this, we propose a concept which utilises a deterministic Non-Monotonic Reasoning methodology to derive user-specified high-level events. We have shown through experimentation that our methodology transforms data with high accuracy when compared to a probabilistic algorithm. Specifically, our methodology recovers the greatest amount of correct events and does not introduce any artificially produced false-positive anomalies.

The remainder of this paper has been organised into the following: Section II will discuss RFID and Non-Monotonic Reasoning. In Section III we will provide an analysis of related work already conducted in this field of study. We propose our methodology for experimentation in Section IV and discuss the experimentation we have conducted on our methodology in Section V. This will be followed directly by our conclusion and future work suggestions in Section VI.

\section{BACKGROUND}

Radio Frequency Identification has been identified as a means to make inefficient tasks such as tracking items both cost-effective and time-saving. Unfortunately, wide acceptance and implementation of RFID systems is still not possible due to the complexities associated with integrating the technology into the environment. Conventional data correcting algorithms may be utilised to help combat these issues associated with RFID in order to enhance the overall integrity of the observations. However, due to the specific complexities associated with RFID, a high level of intelligence such as "NonMonotonic Reasoning - Clausal Logic" must be consulted to take correct courses of action when given ambiguous and misleading information.

\section{A. Radio Frequency Identification}

Radio Frequency Identification (RFID) refers to the wireless technology that relies on radio communication between a tag antenna and reader. It is usually composed of three major components: the tag, the reader and the middleware. The tags have three major classifications as either the battery operated active tags, the semi-active that utilises a battery to enhance the range and the error-prone passive tags that scavenges its power solely from reader's scanning signal. The signals from the tags are collected into the RFID reader which will send out an electro-magnetic scan to detect all antennas within the vicinity. The middleware, also known as the savant, is used as a preliminary defence to filter incoming observations. The information captured by this system is then stored away at an external data warehouse.

Several issues arise due to the nature of the system that hinder the RFID technology from being employed in various applications. These include the low level recordings being made from the tags [1], the errors which arise from the incoming data [2], the large amount of information generated from the incoming data [3] and the complex nature of the spatiotemporal readings [4]. Due to the low amount of information provided from the tags, RFID observational data suffers from allowing any meaningful data to be comprehended without a transformation of the data using related information. During this conversion process, a lack of intelligence will result in misleading information being generated lowering the integrity of all analytical processes performed. 


\section{B. Non-Monotonic Reasoning}

Non-Monotonic Reasoning is a set of logic that arrives at a conclusion using the process of elimination when new information is contributed into the system. Specifically, we are interested in the Clausal Defeasible Logic (CDL) implementation of Non-Monotonic Reasoning due to its easy integration into computational environments and its advantages over other logics such as the team defeat, loop detection and the use of failure-by-looping [5]. There are three levels of ambiguity strength that CDL uses to determine different conclusions:

- $\mu$ : This formula uses only certain information to obtain its conclusion.

- $\pi$ : This formula allows conclusions in which ambiguity is propagated.

- $\beta$ : This formula does not allow any ambiguity to be used to obtain its conclusion.

\section{RELATED WORK}

There have been several methodologies proposed in the past to transform low level raw RFID observations into high level events. Most of these techniques utilise probabilistic methods to infer and derive from partial or erroneous low level observations [6] [7] [8]. While these methodologies work well with enough information, we believe that within scenarios in which there is a large amount of ambiguity present, misleading information may be derived as a result of probabilistic nature. An example of a problem with this system is when an ambiguous partial event may occur such as two people appearing to walk into the same room and the system has identified the two people as having meetings in the past, it will automatically assume that the two people are meeting and will make an event. However, this may not be the case as the people may just be passing by the room or being present in the room without having a meeting.

\section{Methodology}

We propose a concept that utilises an intelligent deterministic methodology to transform low-level raw RFID data into high-level events using Non-Monotonic Reasoning. In the following section, we provide a detailed description of our concept's architecture. This will be followed by the assumptions we have made when designing this system. Finally, we will describe our scenario for this logic engine configuration which we have used for experimentation later on in this study.

\section{A. Architecture}

Our concept conducts the high-level event transformation process within three steps: Analysis, Event Discovery and Event Output. It first analyses the information specifically relating to the user defined events. The information is then passed to the logic engines to determine if an event took place. In the case that an event has been found, our system will output the event allowing a more meaningful data set to be created. All event engines within our research have been tailored to our scenario. However additional logic engines can be defined by the user to accommodate other environments.

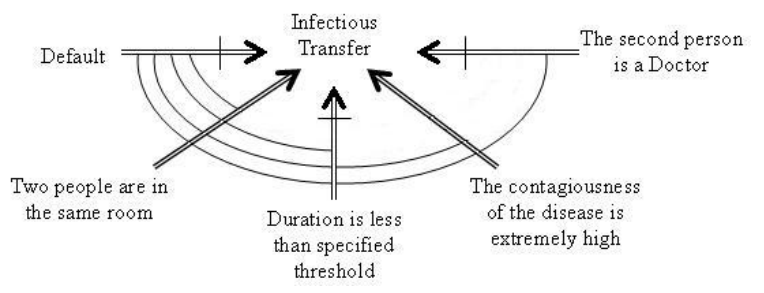

Fig. 1. The Logic Map used for detecting an Infectious Transfer event.

We have created two different logic engines to discover these events; the infectious transfer and the medical examination.

The Infectious Transfer Logic Engine, as shown in Figure 1, has been created with the intent to seek out an event in which it is plausible that a virus may have been transferred. This logic engine has been designed primarily to track a potential outbreak of a disease and will allow doctors to find sources of infections. As such, it works by first examining the records to find an instance if two people are in the same room. If so, it concludes that the event took place. The engine then determines if the time of the event is lower than a userspecified threshold and, if so, it concludes that the event did not take place. It will then decide that the event took place if it is found that the severity of the virus is extremely high (as it is assumed that the higher the severity, the higher the contagiousness). Finally, it checks if the second person in the room is a doctor and, if so, it will conclude that the event did not take place (as it is assumed that the virus will not transfer to a doctor).

The Exam Logic engine has been devised to detect an event in which an examination of patients has been undertaken by a doctor. It has been designed to work to gather information from many various areas such as finding the type of room, the people inside the room, how contagious/severe the disease is and the time spent in that room. From these various fragments of information, the engine then concludes an examination took place if the doctor and patient were in a medical room together. The engine will then decide not to conclude this event if the doctor and patient are in a general room simultaneously. The engine will then check to see if the disease level of the patient is very high or if a transferring event took place and, if so, conclude that an examination did occur. The final set of information which the engine reviews determines whether the duration the doctor and patient spent in the room is less than the designated threshold and, if so, will conclude that the examination event did not take place.

\section{B. Assumptions}

For the Infectious Transfer scenario, we assume that the contagiousness is relative to the severity of the disease and that the doctors cannot catch the virus. For the Examination event, we assume that if a doctor and patient are in a general room for a certain amount of time and the patient's severity of disease is high, the doctor will deliver an examination. Since most middleware is used to eliminate Duplicate RFID readings 


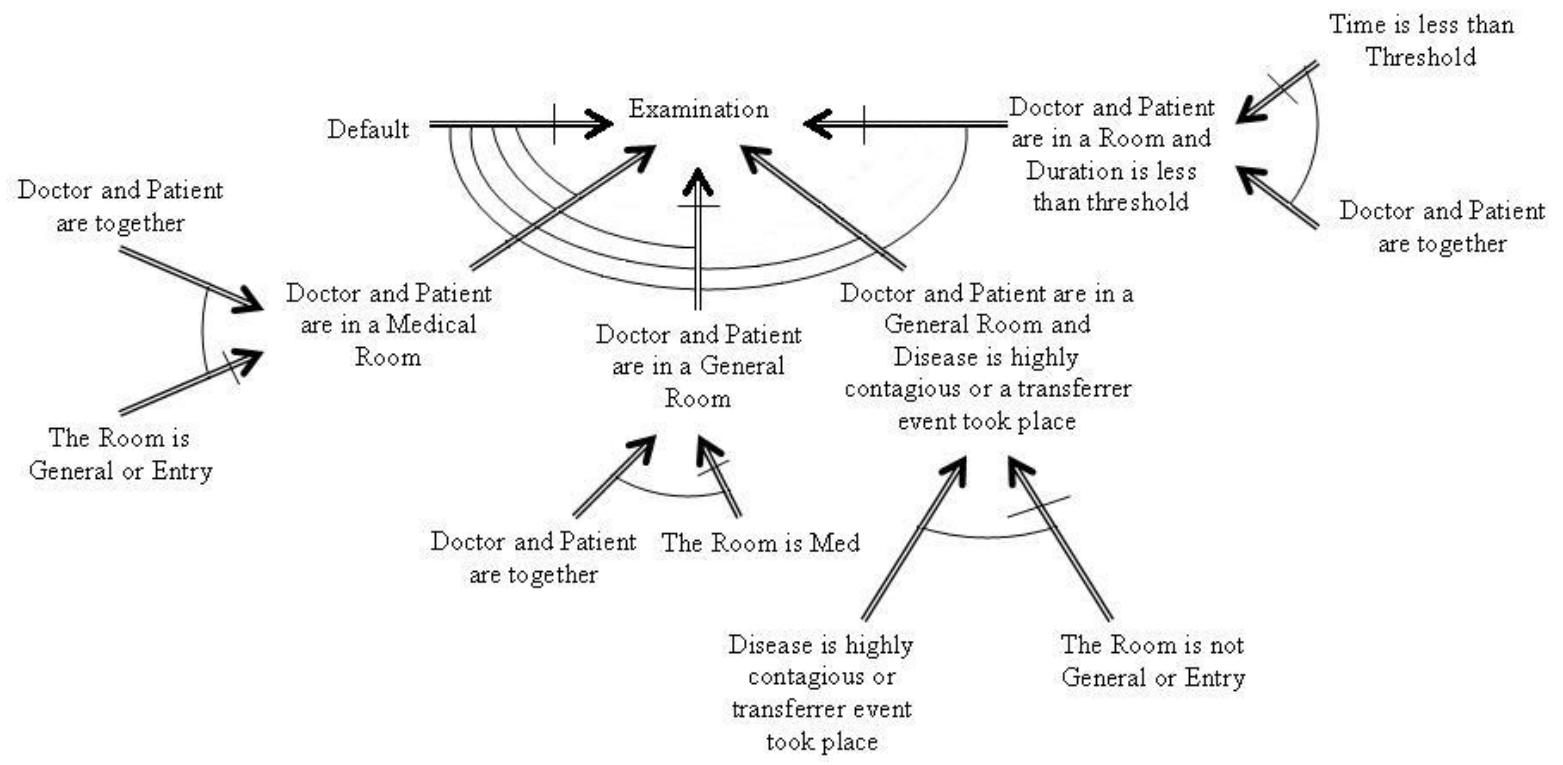

Fig. 2. The Logic Map depicting the detection of an Examination event between a doctor and a patient.

we are assuming that the only errors that persist inside the data sets are that of a missed reading and a wrong read.

\section{Scenario}

The scenario used in this research is based an RFID-enabled hospital such as the Taiwanese medical building implementation [9]. In our simulated scenario, tags are mounted onto bracelets that are worn by hospital patients, staff and doctors to track their movements within the hospital. We also track the movements of hospital staff so that high level events involving interactions between the patients and staff can be recorded and monitored. For this event, we have decided to track three people's movements between the hours of 7:30am and $12 \mathrm{pm}$ - this generates a total of 798 records of observations.

\section{EXPERIMENTAL EVALUATION}

To properly demonstrate the significance of our contribution, we have simulated a scenario in which high-level RFID events may be transformed from low-level raw observations. In the following section we discuss the environment of the computer used for experimentation and the results with analysis derived from our studies.

\section{A. Environment}

Our concept makes use of the Data Model for RFID Applications (DMRA) to store all relevant RFID data [10]. The tables found in DMRA and the procedure language PL/SQL were implemented on Oracle 10g SQL Plus system. The Clausal Defeasible Non-Monotonic Reasoning Engine is written in the $\mathrm{C}$ language, implemented on Cygwin Version 1.5.24-2 and communicates directly with Oracle PL/SQL commands. The computer used for experimentation is is a Microsoft Windows XP machine running Service Pack 3, Intel(R) Pentium(R) 4 CPU $2.79 \mathrm{GHz}$ with $2.00 \mathrm{~GB}$ of RAM.
To extensively experiment our methodology, we considered a simulated hospital scenario that housed a number of potential and ambiguous high-level events that may be transformed. The majority of the relevant information is found within the Observation table, part of the DMRA scheme, however other referential tables have been used to find other characteristics for the logic engines such as people's names, type of person, type of location, etc.

\section{B. Results and Analysis}

To determine if Non-Monotonic Reasoning could be utilised to intelligently discover events, we conducted a trial upon our simulated medical data set to transform the Infectious Transfer and Examination high level events. Additionally, we compared our methodology to a Probabilistic Algorithm also used to determine these events. The experiment was designed to determine if both of the methodologies could accurately determine an event, this is reflected within the Number of Correct Inserts series. The amount of False Negatives and False Positives were also recorded in the experiment to illustrate where each methodology fails to record ambiguous events. Within the findings of these experiments illustrated in Figure 3, it can be surmised that the $\pi$ and $\beta$ formulae detected the most events with very little error. The second best performing algorithm was the Probabilistic Algorithm which achieved $20 \%$ higher correct event detection than anomalous events. The $\mu$ formula obtained the lowest accuracy being that it did not recognise any correct event resulting in it achieving $100 \%$ errors.

From the above results, it can be seen that the $\pi$ and $\beta$ formulae provided the highest amount of accuracy followed by the Probabilistic Algorithm. The reason why the $\pi$ and $\beta$ formulae has achieved a higher integrity level than that of the $\mu$ formula is due to $\mu$ only allowing strict rules to arrive at 


\section{Event Discovering Results}

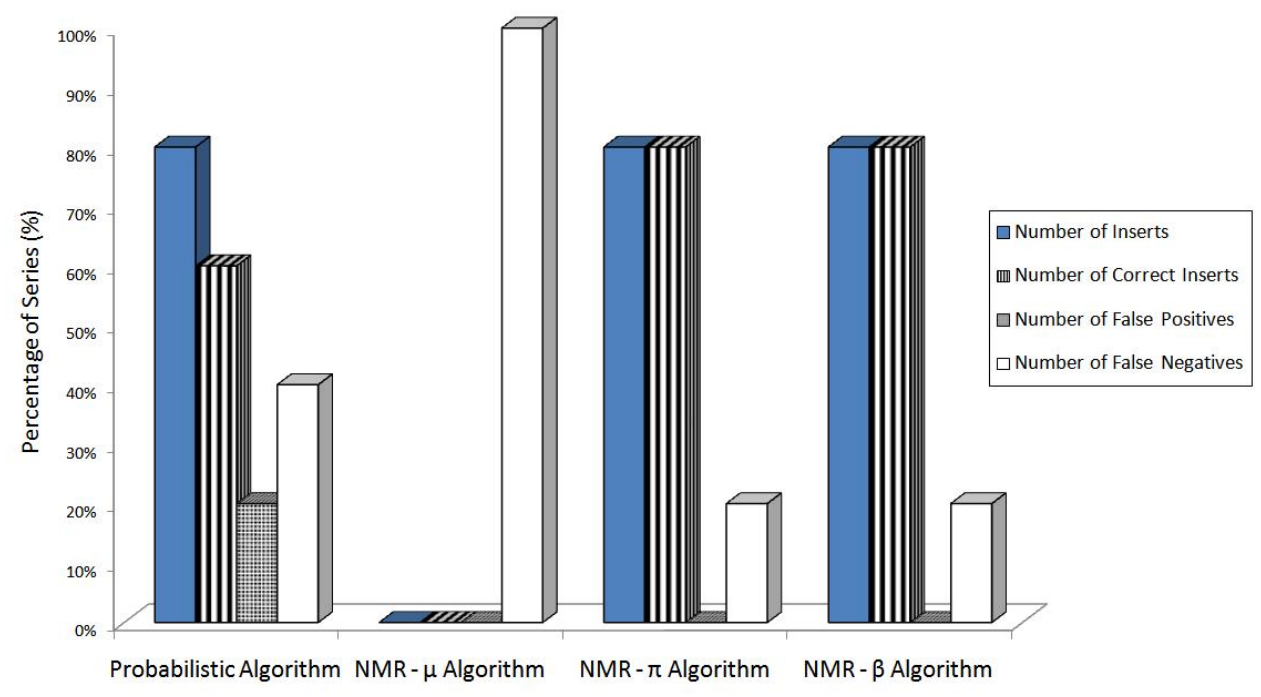

Fig. 3. The results of the Non-Monotonic Reasoning Experiment where the event discovery has been found for the Probabilistic Algorithm and three CDL formulae: $\mu(\mathrm{Mu}), \pi(\mathrm{Pi})$ and $\beta$ (Beta).The different data represented include the number of inserts (solid shaded bar), the number of correct insertions (striped bar), the number of false positives (dotted bar) and number of false negatives (solid white bar).

a conclusion. This will result in the formula not attempting to solve problems that require a certain degree of latitude given to ambiguity unlike $\pi$ and $\beta$. We have found two core strengths while using a deferred Non-Monotonic Reasoning high-level event transformer. The first is that we are able to obtain high level of accuracy when compared to other stateof-the-art probabilistic techniques. The second is that by using a deterministic cleaning approach, there is a reduction in the amount of false positive anomalies introduced via the transformation process. We have also identified weaknesses present within our architecture that we need to improve upon. First and foremost, we recognise that our methodology is extremely dependent on rules and as such, we need to develop a general interface to accommodate many scenarios. Additionally, we have found that the physical environment needs to be set up specifically to allow X-CleLo to function properly.

\section{CONCLUSION}

Radio Frequency Identification (RFID) is hindered from various characteristics that are not easily rectified through conventional means of data integrity algorithms. We believe that it is only through the use of highly intelligent reasoning can the afflictions which are coupled with RFID may be overcome. Specifically, this study has made the following contribution to our field of study:

- Highlighted the importance of high-level event transformations and the problems associated with it.

- Proposed a concept that utilises Clausal Defeasible Logic Non-Monotonic Reasoning to intelligently identify highlevel user defined events.

- Compared our proposed methodology to a state-of-the-art probabilistic methodology and found that our determin- istic approach achieves a higher identification rate.

With regards to future work we would like to generalise our approach to only accommodate the most common events found in various RFID applications such as the union of two or more tags. We believe that this would be most beneficial to the majority of of implementations. We would also like to compare our approach to other high-level classification algorithms such as Bayesian and Neural Networks to determine which classifier obtains the highest identification rate.

\section{REFERENCES}

[1] N. Khoussainova, M. Balazinska, and D. Suciu, "Probabilistic RFID Data Management," UW CSE Technical Report UW-CSE-07-03-01, March 2007.

[2] S. R. Jeffery, M. N. Garofalakis, and M. J. Franklin, "Adaptive Cleaning for RFID Data Streams,” in $V L D B, 2006$, pp. 163-174.

[3] M. Raskino, J. Fenn, and A. Lenden, "Extracting Value From the Massively Connected World of 2015," Gartner Research, Tech. Rep. G00125949, April 2005.

[4] F. Wang and P. Liu, "Temporal Management of RFID Data," in VLDB, 2005, pp. 1128-1139.

[5] D. Billington, "An Introduction to Clausal Defeasible Logic [online]," David Billington's Home Page, Aug 2007, available from: $<$ http://www.cit.gu.edu.au/ db/research.pdf $>$ [Accessed: 3rd July 2008].

[6] D. J. Patterson, L. Liao, D. Fox, and H. A. Kautz, "Inferring high-level behavior from low-level sensors," in Ubicomp, 2003, pp. 73-89.

[7] M. Philipose, K. P. Fishkin, M. Perkawitz, D. J. Patterson, D. Fox, H. Kautz, and D. Hahnel, "Inferring activities from interactions with objects," Pervasive Computing, pp. 10-17, December 2004.

[8] N. Khoussainova, M. Balazinska, and D. Suciu, "PEEX: Extracting Probabilistic Events from RFID Data," in In ICDE, 2008.

[9] C. Swedberg, "Hospital Uses RFID for Surgical Patients," RFID Journal, July 2005, available from: $<$ http://www.rfidjournal.com/article/articleview/1714/1/1/> [Accessed: 5th June 2008].

[10] S. Liu, F. Wang, and P. Liu, "A Temporal RFID Data Model for Querying Physical Objects," TimeCenter, Tech. Rep. TR-88, 2007. 